\title{
The Evolution of Body Heat Conductivity, Skin and Brain Size in Human
}

\author{
A.I. Ibraimov \\ Laboratory of Human Genetics, National Center of Cardiology and Internal Medicine, \\ Togolok Moldo str., 3, Bishkek, 720040, Kyrghyzstan
}

KEYWORDS Body Heat Conductivity. Skin. Brain Size. Q-heterochromatin. C-heterochromatin. Thermoregulation

\begin{abstract}
What is unusual about the human brain is that we are the only largish mammal whose brain size kept pace with our growth in body size. The plausible reason of this phenomenon is his skin, when after having lost its hair it became the largest and almost universal organ of sense, which begins functioning as early as in the prenatal period of human development. It is assumed that $H$. sapiens became the possessor of the largest brain among the primates after he has lost hair on his skin. Apparently skin became hairless as a result of evolution of condensed chromatin in the genome of the direct ancestors of a modern human. In particular in their karyotypes together with chromosomal Q-heterochromatin regions three pairs of autosomes (1,9 and 16), and Y chromosome became the carriers of unusually large C-heterochromatin regions, which have lead to significant increase of body heat conductivity. In conditions of tropical Africa, where our ancestors inhabited, availability of skin covered with hair became a serious obstacle in keeping the temperature homeostasis, particularly in dissipation of excessive heat from the organism that finally has lead to hairlessness. Given this, the heat protection function of the hair cover was taken over by a large amount of subcutaneous adipose tissue.
\end{abstract}

\section{INTRODUCTION}

By 2.4 million years ago new types of hominid had appeared in the fossil record of eastern and southern Africa. About 1.8 million years ago, a new type of early human (Homo erectus) appeared in the fossil record of eastern Africa, a species that was persist in Africa for more than a million years. By about 1 million years ago, $H$. erectus was also present in Asia, and may have spread to southern Europe. By about 400000 years ago, there had been enough changes in certain human populations for a few species of early human to be recognized. This may have marked the emergence of our own species, $H$. sapiens. By 40000 years ago however modern people $-H$. sapience sapience - to have been the sole occupants of the Europe and Asia (Stringer, 1996).

One of the more surprising features of human evolution is that so little change in apparent function took place over two million years. Our brains may have been large but they were certainly not creative (Mithen, 1996). The brain in the Neanderthals, a side branch of human evolution, was actually larger than modern human brains, and we now know that it is unlikely that Neanderthals were our ancestors (Trinkaus, 1994; Krings et al., 1997). And then, certainly more than fifty thousand years ago, and perhaps as much as one hundred thousand to two hundred thousand years ago, a transformation happened. The brain of the humans of the last hundred thousand years have clearly been functioning differently from those of the two and half million or so stable years before at least because technology, art, religion and warfare developed quickly. What happened to bring about the transformation? On the other hand no changes in brain size were associated with the explosive developments in culture, knowledge and social organization over the past one hundred thousand years.

Almost all the "explanations" for recent hominid evolution are variants of social hypothesis relating to the pressures produced by factors such as food gathering, hunting, scavenging, home base behavior, defence against carnivores, and the need to communicate within relatively large social groups (e. g. Aiello and Dunbar, 1993; Foley, 1995; Mithen, 1996; Noble and Davidson, 1996; Rose and Marshall, 1996). These hypotheses almost always include at some point a statement along the lines of "these selective pressures would have driven the evolution of a large and effective brain".

Without arguing for the merits and shortcomings of these hypothesis we would just like to note that possibly in the origin of the modern human such processes as evolution of his body heat conductivity, skin and brain size could be of certain importance. 


\section{FACTS}

\section{Human Brain}

We know much more about the human brain in contrast to what we understand about it. Concerning the issues that are being discussed here the following facts are most important.

The primates as a group have been able to maintain larger brain sizes in relation to body mass than any mammalian group.

Our brain is at least three times as large as that of an ape with the same body size.

During the first year of life the infant's basal metabolic rate (BMR) increases. This increase is associated with the unusually high metabolic demands of rapid growth, particularly with the fast growth of the brain. At this early age the metabolic demands of the brain accounts for more than $50 \%$ of the total BMR of the baby. This is because the brain is an unusually expensive metabolic tissue and in an infant makes up a much larger percentage of total body weight (14\%) than in an adult (2.0-2.7\%). After the first year of life, the growth of the brain slows considerably and the ratio of brain weight to body weight decreases to its adult values (Aiello, 1996).

\section{Human Skin}

Skin is the largest organ in human body, and weighting about $10 \mathrm{~kg}$ in adults its area is more than $1.8 \mathrm{~m}^{2}$. Skin is a large sense organ, separating and at the same time connecting with the outside world, where receptors, which perceive signals from the external and internal environment are located. In skin, to a certain degree of development, there are in germs of all organs of sense. In embryogenesis the skin and brain are formed simultaneously from ectoderm. Skin, organ of secretion (sweat), photochemical and metabolic processes (synthesis of vitamin $\mathrm{D}$, melanin, etc.), respiration and protection (from UV-rays, microbes chemical substances, mechanical injuries, cold, heat and many other things). Skin sensitivity increases with decrease of hair follicle (palms, lips, some part of genitals, sole), i.e. the hairless skin is rather rich in various sensory receptors. Animals, including human beings, in principle can exist without organs of eyesight, hearing, smell and taste, but they cannot exist without the organ of touch.
Many mammals sweat, but human system is the most effective. First, thermal sweat is produced by eccrine glands that pour on to the skin a watery solution with virtually no fats or protein and very little salt. In people, apocrine glands, which produce solutions rich in fats, protein and salts and evaporate more slowly are limited to such areas as the face and hands. Second, human skin is covered by more than 1.5 million sweat glands, which produce copious amounts of sweat over the whole body. Human lose most of their metabolic heat from the body surface, much of it by sweating. An individual can lose more than 2 litres of water in an hour in this way. Third, our lack of body hair also ensures that sweat provides very efficient cooling as it evaporates from heating skin. A man weighting $90 \mathrm{~kg}$ can sweat over 2 litres of water in an hour of normal walking on a hot day in the desert. Active young men will normally lose over 8 litres of water during a day when the desert temperature at midday is above $40{ }^{\circ} \mathrm{C}$. The efficient cooling provided by sweating may have been a significant factors in allowing people to run long distances because the high levels of heat produced through running provide a substantial heat load (Baker, 1996).

Some $1.8 \mathrm{~m}^{2}$ of skin surround an adult body, making skin the largest sense organ. Because it cannot be "shut off", it is a constant state of readiness to receive messages. The first sensory input in life comes from the sense of touch while the fetus is in the womb, and touch continues to be the primary means of experiencing the world through infancy and well into childhood, indeed playing a major sensory role right through to old age. Touch is the first sensory system to develop, and it will continue to function even after sight and hearing have failed. The term touch includes several tactile senses: pain, pressure, temperature and muscle movement. Many sensory receptors at different levels in the skin are responsible for conveying the nerve signals from thermal, mechanical, chemical and electrical stimuli (Field, 2003).

\section{Body Heat Conductivity (BHC) and Condensed Chromatin (CC) in Human Genome}

BHC consists of two components: transfer of thermal energy for short and long distance. The first component, a physical one, is restricted by individual cells, where heat is transferred 
between cytoplasm and nucleus within the cell, or between the neighboring cells in the tissue (Ibraimov, 2003). The second component, a physiological one, represents a long-distance heat transfer from more hot parts of the body to the less hot ones with the help of circulating through the whole organism liquids, such as blood and lymph (Ibraimov, 2004).

There is a significant intra population variability by the human $\mathrm{BHC}$, and it is connected with the amount of chromosomal Q-heterochromatin regions (Q-HRs) in his genome (Ibraimov and Tabaldiev, 2007).

Q-HRs is available only in the genome of three higher primates (H. sapiens, $P$. troglodytes and G. gorilla). C-HRs is available in the genome of all higher eukaryotes, including great apes. However only in the human karyotype there are three pairs of chromosomes with unusually large segments of $\mathrm{C}$-heterochromatin. There are no C-HRs of such size on the chromosomes of chimpanzee and gorilla.

Q- and C-HRs are also available on the Y chromosome of human and gorilla. However the size of constitutive heterochromatin in human Y chromosome is much larger than that in gorilla (Pearson, 1973; Paris Conference, 1971, 1975; Pearson, 1977; Seuànez, 1987). In addition, the long arm of Y chromosome, where Q- and C-HRs are located, demonstrates wide polymor-phism in human population (Ibraimov and Mirrakhimov, 1985). Thus, by the total amount of CC, the human surpasses all other higher primates, as in his genome, in addition to Q-HRs on seven pairs of autosomes $(3,4,13-15,21$ and 22) there are three pairs of autosomes (1,9 and 16) with large C-HRs (Pearson, 1973; Paris Conference, 1971, 1975; Pearson, 1977).

\section{INTERPRETATION OF FACTS}

\section{Human Brain}

When expressed as a percentage of body weight, the human brain is not particularly large or unusual. The squirrel and the human, for example, both have brain which constitute around $2 \%$ by weight of the body (Crawford, 1992). Many small mammals have relatively large brains (Horrobin, 1998). What is unusual about the human brain is that we are the only largish mammal whose brain size kept pace with our growth in body size.
Brain size alone does not determine intelligence and creativity. Among modern humans - and indeed other mammals - there is little evidence of a tight connection between brain size and intelligence. For example, the brain of an elephant is 3-4 times larger than the human one. Neanderthals had brain sizes rather larger than those of modern humans (Trinkaus, 1994) yet it is unlikely that they were more intelligent. Autism is associated with a somewhat larger brain size than normal (Davidovitch et al., 1996).

While brain size is undoubtedly of some importance in that it increases the potential number of neuronal units which may interact, it is clearly not what matters most. The richer are the connections, the richer will be the possibilities for skilled action. The richness of the connections in the context of the brain depends on two main factors the overall connections made by nerve fibre tracts between different brain areas and the richness of the microconnections made between the fine dendritic extensions of the nerve cells. Accurate counts are almost impossible but it is likely that most individual neurons are capable of making between ten thousand and a million individual connections. It is likely to be the richness or otherwise of these microconnections which determines the level at which complex integrated functions can occur (Horrobin, 1998). In this connection Horrobin (1998) assumes that: "in the period fifty thousand to two hundred and fifty thousand years ago something fundamental happened which was so profound that it transformed hominid culture and made us human. My proposal for this is that it was associated with the introduction of increased activity of one or more phospholipases or perhaps cyclo-oxygenases, and that this led to exuberant modelling, pruning and remodelling of synapses with important changes in the development and pruning of microconnections" "Changes in brain metabolism are responsible for changes in brain size and connectivity which led to the emergence of human from our hominid ancestors" ...'In a real sense I suggest that schizophrenia and its associated metabolic variants are what made us human".

Modern humans develop very slowly. They reach sexual maturity late and have a long life. Many people survive past the age of reproduction. We have no idea when this 
distinctive pattern emerged, and some believe it was already present in early hominids. However, new microscopic techniques for examining fossil bones and teeth suggest that it is a recent evolutionary development, which was linked with the growth demands of a large brain as well as the emergence of culture and language. Complex human abilities require a long learning period and also favour the survival of experienced individuals who can pass on their knowledge (Stringer, 1996).

Human are born in an immature state and undergo a relatively longer maturation period than do apes and other primates. This change in developmental timing during hominid evolution was accompanied by a complex of distinctively human patterns, such as increased dependance on learning, enhanced parental care and the defence of a home base. Such a shift in developmental timing has usually been assumed to occur very early in hominid evolution. However, recent refinements in analysing tooth development indicate that the dentitions of hominids earlier than $H$. erectus have developmental rates characteristic of apes rather than humans. An extended period of infant maturation with all its implications for social behaviour may hence have evolved later than was previously believed (Potts, 1996).

Between the appearance of $H$. erectus and $H$. sapiens, around 400000 years ago, human manipulative skills changed only gradually. Human spread into seasonal climates such as subtropical Africa and Asia, and subsequently into temperate Eurasia, by which time fire had also been controlled. These technological changes probably reflect an increased human ability to cope with harsher more seasonal environments. Interestingly, this later geographic expansion was associated with a increase by a third in the size of the human brain (Trinkaus, 1996).

Several problems remain even after $H$. sapiens entered the scene, f.e., hominids that were ruggedly built but basically modern in their anatomy appeared between 130000 and 100000 years ago, but the first clear indication of fully modern behavioural capabilities is not found until much later. Speech and language may, perhaps, have appeared as early as 100000 years ago (Potts, 1996).

If we can understand some of the factors which determine the brain size and connectivity in the present day, it is possible that these will give us clues to the factors which influenced these features over the past three million years.

\section{Human Skin}

We assume that namely the skin has led to the formation of many more abundant microconnections and also to parts of the brain being connected which had not been connected before. It would also lead to changes the rates of dendritic pruning during development and puberty, which are also important in determining the connectivity of the adult brain.

Size alone of the brain is important in providing enough neuronal elements to interact to produce a complex network. But it is the richness and specifity of the fine connections of that network which determine the complexity of the information processing which can occur (Horrobin, 1998).

Information from touch-sensitive nerve cells ultimately crosses the sensory cortex to the opposite side of the brain where it is processed. The amount of space needed by the cortex is related not to the size of the body part but to the nerve density: areas with more nerve endings, such as fingertips, tips and genitals, require more space in the cortex than the back, which has fewer nerve endings (Schiffman, 1990).

Young children depend on touch for learning about the world including the quantities of temperature, texture, shape, softness, sharpness, elasticity and resilience. Children also learn safety from touch such as avoiding stoves, sharp objects or frostbite, and they may learn how to write through touch if given hand-over-hand assistance in handwriting classes. Touch is not only critical for growth, development, communication and learning but also serves for comfort, reassurance and self-esteem (Field, 2003). In the words of Schanberg: "Touch is ten times stronger than verbal or emotional contact, and it affects damned near everything we do. No other sense can arouse you like touch. We always knew that, but we never realized that it had a biological basis. If touch did not feel good, there would be no species, parenthood or survival. ... We forget that touch is not only basic to our species but the key to it" (Schanberg, 1995). The role of touch in culture, health and caring for infant is well known. 


\section{Human BHC and CC}

According to Seuánez (1987), the cytogenetic and molecular evolutionary events may have bean independent. Thus a change in only a small fraction of the genes could contribute to a genomic revolution (Cronin and Sarich, 1976).

Also, true revolution may be found at the chromosomal level. Chromosomal rates of evolution seem to correlate with the degree of morphological evolution and rates of speciation (Wilson et al., 1977). Such a change would be macrogenic and would best be sought at the level of satellite DNA, quantity of DNA per locus, chromosomal rearrangements and regulatory loci mutations (White, 1998).

The evolutionary studies carried out so far seem to indicate that the euchromatic regions of the chromosomes in the different species of primates analyzed are quite similar (Dutrillaux, 1979; Dutrillaux et al., 1981). The main differences in these species are due to the different amounts and localization of heterochromatin (Clemente et al., 1990). These data are in agreement with those of Dutrillaux et al. (1981) according to which heterochromatin is not distributed at random in the chromosomes, but is usually found in the same regions, depending on the species or genera studied.

The unique features of the human karyotype include a fusion of two chromosome pairs (reducing the diploid number to $2 \mathrm{n}=46$ ), and the emergence of $\mathrm{C}$-banded regions below the centromeres of chromosomes 1, 9 and 16, and on the long arm of the Y chromosome (Paris Conference, 1971, 1975).

For unknown as yet reasons, at late stages of the evolution of life, in ancestor of contemporary three higher primates (H. sapiens, P. troglodytes and $G$. gorilla) there appeared a new type of constitutive heterochromatin - "Q-heterochromatin". Thus, one can say with certainly that Q-heterochromatin originated in tropical Africa. But the main difference between these species is the presence of wide quantitative variability of chromosomal Q-HRs only in the genome of human populations (Pearson, 1973; Paris Conference, 1971, 1975; Pearson, 1977; Ibraimov and Mirrakhimov, 1985; Seuànez, 1987; Ibraimov and Tabaldiev, 2007).

At present we have the data that the constitutive heterochromatin constitutes the structural basis of the physical component of the human BHC (Ibraimov, 2003; 2004; Ibraimov and Tabaldiev, 2007). Thus we assume that because of great amount of constitutive heterochromatin in human genome in comparison with other higher primates, the human body became to be distinguished by the greatest heat conductivity. In addition, this important circumstance has lead to appearance of hairless skin.

\section{CONCLUSION}

“Man's naked skin could not been produced by natural selection" A. Wallace

One hardly can imagine an animal which high intellectual would be disadvantage for it. However only a man, the youngest of the known living creatures on the Earth possesses such an exclusive feature. What is the basis for having such a precious gift?

We believe that the increase of the human brain size was not the result of drastical changes of the structural genes. Most likely it was the consequence of more ordinary events, such as evolution of constitutive heterochromatin in chromosomes, BHC and skin.

In the heterochromatin part of genome in the direct ancestors of modern human some changes occurred about 100-150 000 years ago; in addition to Q-HRs, on three pairs of autosomes (1,9 and 16), and on the $\mathrm{Y}$ chromosome unusually large $\mathrm{C}$ - heterochromatin regions appeared, which do not exist in karyotypes of chimpanzee and gorilla. Thus by the total amount of $\mathrm{CC}$ the genome of the $H$. sapiens turned out to be richest one.

As we have demonstrated before, the amount of chromosomal Q-HRs in genome is connected with the human body heat conductivity (Ibraimov and Tabaldiev, 2006).

We assume that assemblage of the greatest amount of $\mathrm{CC}$ in the $H$. sapiens genome among the higher primates was the turning point in human evolution, as exactly this circumstance has lead to disappearance of hairy cover on his skin. The latter turned out to be the main factor responsible for increase of the brain size during the first years of life of the H. s. sapiens.

Naked skin was a result of long series of events, each depending on the other, and each unpredictable and unique. Apparently, the main reasons for appearance of hairless skin were the following factors:

1) increase of BHC because of high Q-HRs 
and C-HRs content in the genome of the direct ancestor of modern human;

2) quantitative and qualitative changes of the diet composition (Crawford, 1992; Rose and Marshall, 1996; Horrobin, 1998), which lead to increase of heat production in the organism demanding efficient heat loss from the body for preservation of temperature homeostasis;

3) tropical climate of Africa, where the ancestors of the $H$. s. sapiens inhabited, had a strong selective influence on such organisms because their bodies have changed towards high heat conductivity and heat production. It is possible that in such conditions the best solution of the thermoregulation problems was modification of skin: loss of hairy cover, increase of its heat dissipation ability by increase of the amount of the eccrine glands, blood vessels, and other changes. Given this, the heat-protection function of hair passed over to the layer of subcutaneous fat. Adipose tissue also provides us with defenses against starvation, which could have represented major competitive advantages of many stages of our evolutionary history. Such skin, in addition provided with a great amount of sensory receptors, cannot but influence the postnatal development of the brain size because they have a close ontogenetic connection, since as in early embryogenesis the skin and brain are formed simultaneously from ectoderm.

We also assume that in formation of structure, and especially in functioning of human hand an important role could be played by this hairless skin. For example, it is known that the human brain significantly differs from the brain of animals by the content of melanin in substantia nigra. It serves as a center of plastic tonus that has developed as an auxiliary instrument at the motor centres (Sepp, 1949). The content of melaninlike pigment is the highest in the substantia nigra of predators, apes, and is of the highest degree in human - possessor of the most highly differentiated movements. It is notable that melanin appears in the brain of an infant only at the third year of age.

The facts of simultaneous formation of skin and brain at the 9-12 ${ }^{\text {th }}$ weeks of embryogenesis, localization of melanin synthesizing system in skin and substantia nigra, and recent appearance of hairless skin before formation of the secondary signal system about 40 - 50000 years ago unambiguously testifies to a certain role of naked skin in evolution of human brain.
Without going into further detail we'll cite several statements, which belong to the specialists who have been speculating on the role of skin in the adaptive evolution of a man for a long time: "Open, not protected by anything man's skin is a principally important phenomenon in the "hairy kingdom" of higher animals. It, as any cover of any living being, ensures the integrity of the organism and serves as communication facilities with the surrounding world. Such communication is performed by numerous receptors penetrating the skin and conveying information on the state of the external and internal environment in the brain .... Loss of hair on the palms and modification of wrist, which was connected with this, was a necessary condition for beginning of the activity with the use of instruments by our remote ancestors. In human the importance of the palm skin significantly increased. By amount and complexity of skin receptors the human hand ranks first. Development of such complex receptor system in the hand skin accompanied by corresponding development of the analyzing brain system, and consequently by the increase of human creative abilities" (Malenkov and Kovalev, 1986); "in the process of evolution skin ceases to be only the barrier-protective organ, it also becomes the organ of sense" (Frolov, 1982); "formation of skin as a photochemical and metabolic organ may be related to the time when the human labor activity became more sophisticated, i.e. to the time of transition from paleo-anthropos to neo-anthropos" (Kovalev et al., 1983).

Finally, we believe that loss of hair was beneficial for human in the sense that if he were not naked, he would not begin to manufacture clothes, which is impossible without availability of fine instruments like awl, needle, and other instruments, production of which is connected with fine coordination of hands' movements. Fire striking and dwelling construction is also impossible without skillful hands even with availability of high Intellect. " Naked skin made the human not only improve his labor, it also promoted the brain development in another direction. It is possible to say that with appearance of skin a vast area of body has been formed provided with highly differentiated and fine receptors of different signals both from the outer and inner environment. It required a relevant development of the analyzing brain 
systems. Brain function became more complex, and brain function started to develop" (Malenkov and Kovalev, 1986). That is as the folk say: "Every cloud has its silver lining". A human, instead of his hair that he has lost, acquired clothes, which is more important in sense that in addition to body protection from cold (physiology) it has an important ethnical and cultural, psycho-emotional, social and economic importance that promotes the scientific and technical progress. In addition the latter facilitates functioning of the temperature homeostasis in human. Therefore in reply to the old question, namely: "has a human survived because he became intelligent, or, he became intelligent only because he managed to survive", we have chosen the second version (Ibraimov, 1993).

The common ancestor of humans and apes was a tropical creature, and members of the human family did not spread out of the tropics and subtropics until about a million years ago. Today there are 6000 million of us, found all over the earth's surface. The human species can tolerate such a range because each of us carries a tropical microenvironment in the form of clothes and dwellings (tropicalization).

We assume that the apes could not leave the boundaries of tropical Africa as they were not able to tropicalize the microenvironment around their bodies, and their hair cover was not sufficient enough to protect the organism of these heat-loving animals even in conditions of Africa savanna climate.

The modern pattern of bipedal locomotion has a long and complex evolutionary history. There are many theories of the emergence of this unique pattern of locomotion. Without disputing these theories we would only like to underline a possible role of the special form of our hands in development of bipedality. Before the first species of Homo appeared some 2 or more million years ago, the australopithecines were bipeds. It is possible that they could walk but not run, and were probably active climbers. In other words, after the direct ancestors of the H. sapiens lost their body hair they started to pass over to fully erect walking.

We suppose that our hands have acquired the contemporary features after the ancestors of the $H$. sapiens had lost their hair to solve the problem of withdrawing surplus heat from the organism, coming across with necessity of body tropicalization in constantly changing conditions of the Africa savanna climate. In this connection one should keep in mind that humans are not very widely flexible in physiological terms. Humans tend to tropicalize their environment virtually everywhere they go. They do this by hands for the most part (clothing, tools, buildings, heating). Thus they were forced to constantly lean on the hind legs though when running they lost speed. As a matter of fact even for modern human the bipedality is not of inherent nature, as, for example, the ability to crawl on all fours. For erect walking an infant needs compulsory and long-term training by the adults. We assume that, apparently, transformation of the frontal extremities into skilful hands, bipedality and erect walking are connected with appearance of hairless skin. All the above, in its turn and to a variable degree they have an influence on the human brain size. Thus the whole evolution of the BHC, skin and brain size is a result of adaptation to constantly changing temperature conditions of the environment.

Schematically the evolution of the BHC, skin and brain size may be roughly presented as follows: in the genome of one of the branches of the hominid together with Q-HRs large segments of C-HRs on three autosomes and Y chromosome appear $\rightarrow$ in such organisms the BHC significantly increases $\rightarrow$ problems appear with the heat loss from the body in conditions of tropical heat and heat protection of the organism when the environmental temperature decreases $\rightarrow$ in such conditions in order to keep the temperature homeostasis, the organisms with high $\mathrm{BHC}$ were deprived of hair for improving the heat loss, and under new skin a layer of subcutaneous fat for heat protection instead of the hair cover was formed $\rightarrow$ gradually the hairless skin has transformed into the largest sense organ $\rightarrow$ with falling of temperature of the African savanna the need in tropicalization of the microenvironment around the body started to increase that is impossible without a skillful hand $\rightarrow$ with switching the frontal extremities for the needs of tropicalization the bipedal locomotion became the bares necessity $\rightarrow$ naked skin with a great amount of the different nerve receptors, skillful hands and bipedal locomotion have promoted, and is being promoting the postnatal increase of the human brain size.

Finally, taking the risk of being laughed out by the colleagues, nevertheless I would presume to express another seditious idea: the increased 
brain, and particularly the naked skin itself have resulted in appearance of a qualitatively new, unknown in world of animals feeling - a sense of shame. Who knows, may be the first leaf covering the genitals of the primitive human was the first herald of future moral principles, which laid the foundation of human community.

Of course it is impossible to test our hypothesis in detail. However it is quite possible to verify some of its key points by experimental research both at the cellular and organism level. At the cellular level it is possible to examine the influence of condensed chromatin in the genome on the cellular thermoregulation; namely, in the cells culture of human and two other great apes to compare the speed of transfer of heat between the cytoplasm and nucleus at a change of temperature in the thermostat above or below $37^{\circ} \mathrm{C}$ (Ibraimov, 2003). At the organism level it is possible to compare the $\mathrm{BHC}$ of these three higher primates, as we have done it in the human populations (Ibraimov and Tabaldiev, 2007).

\section{ACKNOWLEDGEMENTS}

I apologize to those authors, whose works were not cited, or were cited only through reviews, owing to space limitations. I am grateful to Dr. S. Tabaldiev for excellent technical assistance.

\section{REFERENCES}

Aiello, L.C. and Dunbar, R.I.M.: Neocortex size, group size, and the evolution of language. Curr. Anthropol., 34: 184-193 (1993).

Aiello, L.C.: Body size and energy requirements, pp. 4145, In: The Cambridge Encyclopedia of Human Evolution. S. Jones, R. Martin and D. Pilbeam (Eds.). Cambridge University Press, Cambridge (1996).

Baker, P.T.: Human adaptations to the physical environments, pp 46-51, In: The Cambridge Encyclopedia of Human Evolution. S. Jones, R. Martin and D. Pilbeam (Eds.). Cambridge University Press, Cambridge (1996).

Clemente, I.G., Ponsa, M., Garcia, M. et al.: Evolution of the simmiformes and the phylogeny of human chromosomes. Hum. Genet., 84: $493-506$ (1990).

Crawford, M.A.: The role of dietary fats in biology: their place in the evolution of the human brain. Nutr. Rev., 50: 3-11 (1992).

Cronin, J.E. and Sarich, U.M.: Molecular evidence for dual origin of mangabeys among old world monkeys. Nature, 260: 700-702 (1976).

Davidovitch, M., Patterson, B. and Gartside, P.: Head circumference measurements in children with autism. J. Child. Neurol., 11: 389-393 (1996).
Dutrillaux, B., Counturier, J. and Viegas-Pèquignot, E.: Chromosomal evolution in primates, pp 176-191, In: Chromosomes Today, vol 7. M.D. Bennet, M. Boboraw and G.M. Herwitt (Eds.). New-York (1981).

Dutrillaux, B.: Chromosomal evolution in primates: tentative phylogeny from Microcebus murinus (Prosimian) to man. Hum. Genet., 48: 251-314 (1979).

Field, T.: Touch. MIT Press, Cambridge, Mass (2003).

Foley, R.A.: Causes and consequences in human evolution. J. R. Anthropol. Soc., 1: 67-86 (1995).

Frolov, E.P.: Kozha kak organ chuvstv, pp. 168-197, In: Kozha (stroenie, funktsiya, obschaya patologiya $i$ terapiya), (in Russian). Meditsina, Moskva (1982).

Horrobin, D.F.: Schizophrenia: the illness that made us human. Medical Hypotheses, 50: 269-288 (1998).

Ibraimov, A.I.: Condensed chromatin and cell thermoregulation. Complexus, 1: 164-170 (2003).

Ibraimov, A.I.: The Origin of Condensed Chromatin, Cell Thermoregulation and Multicellularity. Complexus, 2: 23-34 (2004)

Ibraimov, A.I.: The origin of modern humans: A cytogenetic model. Hum. Evol., 8: 81-91 (1993).

Ibraimov, A.I. and Mirrakhimov, M.M.: Q-band polymorphism in the autosomes and the $\mathrm{Y}$ chromosome in human populations, pp. 213-87, In: Progress and Topics in Cytogenetics. The $Y$ chromosome. Part A. Basic Characteristics of the $Y$ chromosome. A. A. Sandberg (Ed.). Alan R. Liss Inc, New York (1985).

Ibraimov, A.I. and Tabaldiev, S.K.: Condensed chromatin, cell thermoregulation and human body heat conductivity. J. Hum. Ecol., 21(1): 1-22 (2007).

Kovalev, I.E., Kovalev, E.N. and Tavintsev, V.D.: Gipoteza molekulyarnogo mehanizma zabolevaniya rasseyannym sklerozom, pp. 77-79, In: Ispol'zovaniya modelei patologicheskih sostoyanii pri poiske biologicheski aktivnyh soedinenii ch. 1 . (in Russian) Moskva (1983).

Krings, M., Stone, A. and Paabo, S.: Neanderthal DNA sequences and the origin of modern humans. Cell, 90: $19-30$ (1997)

Malenkov, A.G. and Kovalev, I.K.: Kozha i proishozhdenie cheloveka. (in Russian) Priroda, 6: 76-83 (1986).

Mithen, S.: The Prehistory of the Mind: A Search for the Origins of Art, Religion and Science. Thames and Hudson, London (1996).

Noble, W. and Davidson, I.: Human Evolution: Language and Mind. Cambridge University Press, Cambridge (1996).

Paris Conference, 1971, and Supplement, 1975. Standartization in Human Cytogenetics . Birth Defects, XI: 1-84.

Pearson, P.L.: Banding patterns chromosome polymorphism and primate evolution. Prog. Med. Genet., 2: 174-197 (1973).

Pearson, P.L.: The uniqueness of the human karyotype, In: Chromosome Identification: Technique and Applications in Biology and Medicine. T. Caspersson and L. Zech (Eds). Academic Press, New York (1977).

Potts, R.: The hominid way of life, pp. 325-334 In: The Cambridge Encyclopedia of Human evolution. S. 
Jones, R. Martin and D. Pilbeam (Eds.). Cambridge University Press, Cambridge (1996).

Rose, L. and Marshall, F.: Meat eating, hominid sociality, and home bases revisited. Cure. Anthropol., 37: 307338 (1996).

Schanberg, S.: The genetic basis for touch effects, pp. 67-69, In: Touch in Early Development. T. Field (Ed.). Erlbaum, Mahwah (1995).

Schiffman, H.R.: Sensation and Perception: An integrated Approach. Wiley, New-York (1990).

Sepp, E.K.: Istoriya razvitiya nervnoi sistemy pozvonochnyh. (in Russian) Moskva (1949).

Seuànez, H.N.: The chromosomes of man: evolutionary considerations, pp 65-89, In: Cytogenetics. G. Obe and A. Baster (Eds.). Springer, Berlin (1987).

Stringer, C.B.: Evolution of early humans, pp. 241-251, In: The Cambridge Encyclopedia of Human Evolution. S. Jones, R. Martin and D. Pilbeam (Eds.). Cambridge University Press, Cambridge (1996).

Trinkaus, E.: The Neanderthals. Pimlico, London (1994).

Trinkaus E 1996. Evolution of human manipulation. In S Jones, R Martin, D Pilbeam (Eds): The Cambridge Encyclopedia of Human evolution. Cambridge, Cambridge University Press, pp 346-349.

White, M.J.D.: Models of Speciation. Freeman, San Francisco (1998).

Wilson, A.C., Carlson, S.S. and White, T.J.: Biochemical evolution. Annu. Rev. Biochem., 46: 573-693 (1977). 